Document downloaded from:

http://hdl.handle.net/10251/147414

This paper must be cited as:

Piles, M.; García-Tomas, M.; Rafel, O.; Ibañez Escriche, N.; Ramon, J.; Varona, L. (2007). Individual efficiency for the use of feed resources in rabbits. Journal of Animal Science. 85(11):2846-2853. https://doi.org/10.2527/jas.2006-218

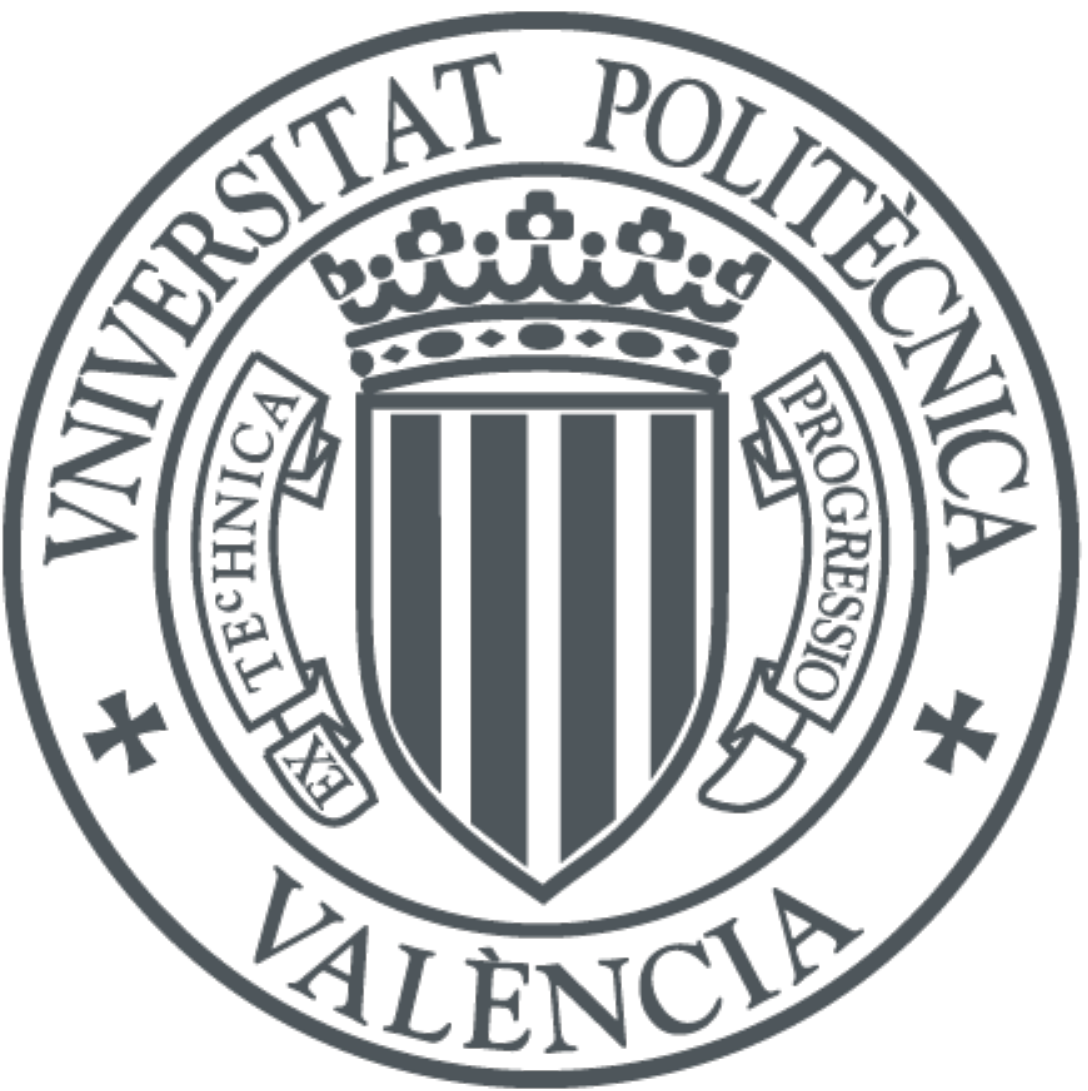

The final publication is available at

https://doi.org/10.2527/jas.2006-218

Copyright American Society of Animal Science

Additional Information 


\title{
Individual efficiency for the use of feed resources in rabbits ${ }^{1}$
}

\author{
M. Piles, *2 M. García-Tomás,* O. Rafel,* J. Ramon,* N. Ibañez-Escriche, $\dagger$ and L. Varona $\dagger$ \\ *IRTA - Unitat de Cunicultura, Torre Marimón s/n., 08140 Caldes de Montbuí, Barcelona, Spain; and \\ $\dagger$ † Area de Producció Animal, Centre UdL - IRTA, C/ Rovira Roure 177, 25198 Lleida, Spain
}

\begin{abstract}
A Bayesian procedure, which allows consideration of the individual variation in the feed resource allocation pattern, is described and implemented in 2 sire lines of rabbit (Caldes and R). The procedure is based on a hierarchical Bayesian scheme, where the first stage of the model consists of a multiple regression model of feed intake on metabolic BW and BW gain. In a second stage, an animal model was assumed including batch, parity order, litter size, and common environmental litter effects. Animals were reared during the fattening period (from weaning at $32 \mathrm{~d}$ of age to $60 \mathrm{~d}$ of age) in individual cages on an experimental farm, and were fed ad libitum with a commercial diet. Body weight (g) and cumulative feed intake $(\mathrm{g})$ were recorded weekly. Individual BW gain (g) and average $\mathrm{BW}(\mathrm{ABW}, \mathrm{g})$ were calculated from these data for each 7-d period. Metabolic BW $\left(\mathrm{g}^{0.75}\right)$ was esti-
\end{abstract}

mated as $A B W^{0.75}$. The number of animals actually measured was 444 and 445 in the Caldes and $R$ lines, respectively. Marginal posterior distributions of the genetic parameters were obtained by Gibbs sampling. Posterior means (posterior SD) for heritabilities for partial coefficients of regression of feed intake on metabolic BW and feed intake on BW gain were estimated to be 0.35 $(0.17)$ and $0.40(0.17)$, respectively, in the Caldes line and $0.26(0.19)$ and $0.27(0.14)$, respectively, in line $R$. The estimated posterior means (posterior SD) for the proportion of the phenotypic variance due to common litter environmental effects of the same coefficients of regression were respectively, $0.39(0.14)$ and $0.28(0.13)$ in the Caldes line and $0.44(0.22)$ and $0.49(0.14)$ in line $R$. These results suggest that efficiency of use of feed resources could be improved by including these coefficients in an index of selection.

Key words: Bayesian analysis, feed efficiency, rabbit, selection

(2007 American Society of Animal Science. All rights reserved.

J. Anim. Sci. 2007. 85:2846-2853 doi:10.2527/jas.2006-218

\section{INTRODUCTION}

In rabbits, current selection programs are focused on selecting paternal lines for growth rate during the fattening period or weight at a fixed age. One objective of selection is to improve feed efficiency as a correlated response to selection for those traits. Feed efficiency is usually measured using the feed conversion ratio (feed intake/weight gain), and is considered to be 1 of the most important traits in rabbit breeding (Armero and Blasco, 1992). However, Piles et al. (2004) found that the genetic correlation between growth rate and feed conversion ratio is not very large, and suggested

\footnotetext{
${ }^{1}$ Acknowledgments: Research was supported by INIA SC00-011. The authors acknowledge comments and suggestions made by M. Baselga and A. Blasco from the Universidad Politécnica de Valencia (Spain) and R. Rekaya for his assistance in solving numerical problems.

${ }^{2}$ Corresponding author: miriam.piles@irta.es

Received April 6, 2006.

Accepted July 19, 2007
}

that other alternatives, such as a selection index, should be used instead, with the objective of improving response to selection for the feed conversion ratio.

On the other hand, Rauw et al. $(2000,2002)$ have described a 2-step procedure that allows comparison of genetic lines by the efficiency of metabolic resource allocation for BW maintenance, growth, and other metabolic processes, such as physiological activity and responses to stress or pathogens.

The aim of the present paper was to describe and implement a Bayesian method based on this strategy using a hierarchical Bayesian scheme (Wakefield et al., 1994). The procedure allowed consideration of the individual variation in the resource allocation pattern, and as a consequence, its possible utilization in an index of selection for feed efficiency (Blasco, 2001). The procedure also had all of the advantages of linear or nonlinear hierarchical models described by Varona et al. (1997) and illustrated later on in cattle (Varona et al., 1998; Rekaya et al., 2000, 2001), poultry (MignonGastreau et al., 2000), and rabbits (Blasco et al., 2003; Piles et al., 2003). 


\section{MATERIALS AND METHODS}

The research protocol was approved by the animal care and use committee of Institut de Recerca i Tecnologia Agroalimentàries (IRTA).

\section{Method}

The model was based on the hierarchical Bayesian scheme of Wakefield et al. (1994). The joint posterior density of an unknown vector of parameters $(\boldsymbol{\theta})$ and hyper parameters $(\phi)$, given the data $(\mathbf{y})$, is expressed as

$$
p(\boldsymbol{\theta}, \phi \mid \mathbf{y}) \propto p(\mathbf{y} \mid \boldsymbol{\theta}) p(\boldsymbol{\theta} \mid \phi) p(\phi),
$$

where $p(\boldsymbol{\theta}, \phi \mid \mathbf{y})$ is the posterior density, $p(\mathbf{y} \mid \boldsymbol{\theta})$ is the likelihood (first-stage of the hierarchical model), $p(\boldsymbol{\theta} \mid$ $\phi)$ is the prior density of the parameters given the hyper parameters (second-stage), and $p(\phi)$ is the prior density of the hyper parameters. In this model, uncertainty about unknowns at some level is accounted for when inferring unknowns at other levels and all (co)variances between observations are taken into account, leading to a correct statement of precision of estimates (Varona et al., 1997).

First Stage of the Model. The first stage of the model defines the multivariate regression model for feed consumption. Individual feed intake in period $j$ was assumed to follow the model: $y_{i j}=a_{i}+b_{i} \times$ $A B W_{i j}^{0.75}+d_{i} \times B W G_{i j}+\varepsilon_{i j}$, where $y_{i j}$ is the cumulative feed intake of individual $i$ during the 7 -d period $j, a_{i}$ is the individual intercept, $b_{i}$ is the individual partial coefficient of regression of feed intake $(\mathrm{g} / 7-\mathrm{d})$ on metabolic BW ( $\left.\mathrm{g}^{0.75} / 7-\mathrm{d}\right), d_{i}$ is the individual partial coefficient of regression of feed intake on BW gain $(\mathrm{g} / 7-\mathrm{d})$, and $A B W_{i j}$ and $B W G_{i j}$ are the average BW (calculated as the average of the initial and final BW) and BW gain of individual $i$ during period $j$. Individual metabolic BW in each period $\left(\mathbf{M B W} \mathbf{W}_{\mathbf{i j}}\right)$ was estimated as $A B W_{i j}^{0.75}$. The $\varepsilon_{i j}$ is the residual.

We assumed that the data were conditionally independent and identically normal distributed. We also assumed that all animals had the same residual variance $\left(\sigma_{\varepsilon}^{2}\right)$ at any period $j$, but more complete models considering residuals to be heteroscedastic or not independently distributed, or both, could also be implemented: $y_{i j} \mid a_{i}, b_{i}, d_{i}, \sigma_{\varepsilon}^{2} \sim N\left(a_{i}+b_{i} \times A B W_{i j}^{0.75}+d_{i} \times\right.$ $\left.B W G_{i j}, \sigma_{\varepsilon}^{2}\right)$. Thus, the likelihood could be written as

$$
\begin{gathered}
f\left(\mathbf{y} \mid \boldsymbol{\theta}, \sigma_{\varepsilon}^{2}\right)=\prod_{i=1}^{N} \prod_{j=1}^{n_{i}} \frac{1}{\sqrt{2 \pi} \sigma_{\varepsilon}} \\
\exp \left\{-\frac{\left(y_{i j}-a_{i}-b_{i} \times A B W_{i j}^{0.75}-d_{i} \times B W G_{i j}\right)^{2}}{2 \sigma_{\varepsilon}^{2}}\right\},
\end{gathered}
$$

where $\mathbf{y}$ is the vector of data of individual feed intake $(\mathrm{g} / 7 \mathrm{~d}) ; \boldsymbol{\theta}^{\prime}=\left\{\mathbf{a}^{\prime}, \mathbf{b}^{\prime}, \mathbf{c}^{\prime}\right\}$ is the vector of parameters of the multivariate regression model; $\mathbf{a}^{\prime}=\left\{a_{i}, i=1, \ldots, N\right\}$ is the vector of individual intercepts; $\mathbf{b}^{\prime}=\left\{b_{i}, i=1, \ldots, N\right\}$; and $\mathbf{d}^{\prime}=\left\{\mathrm{d}_{\mathrm{i}}, \mathrm{i}=1, \ldots, \mathrm{N}\right\}$ are the vectors of individual partial coefficients of regression of feed intake on metabolic BW and BW gain, respectively; $\mathrm{N}$ is the number of individuals with data; and $\mathrm{n}_{\mathrm{i}}$ is the number of records for animal i.

Second Stage of the Model. The second stage of the model describes the interindividual variation of the nonobservable traits: the intercept and both coefficients of regression. Thus, it constitutes the prior information of the parameters of the model in the first stage. These traits were determined by a vector of systematic effects, a common litter environmental effect, an individual additive genetic effect, and a random environmental component that we assumed to be normally distributed. Thus,

$$
\begin{aligned}
\mathbf{a}, \mathbf{b}, \mathbf{d} \mid \boldsymbol{\beta}_{a}, \boldsymbol{\beta}_{b}, \boldsymbol{\beta}_{d}, \mathbf{c}_{a}, \mathbf{c}_{b}, \mathbf{c}_{d}, \mathbf{u}_{a}, \mathbf{u}_{b}, \mathbf{u}_{d}, \mathbf{C}, \mathbf{G}, \mathbf{R} \\
\sim \mathrm{N}\left(\begin{array}{l}
\mathbf{X} \boldsymbol{\beta}_{a}+\mathbf{W} \mathbf{c}_{a}+\mathbf{Z} \mathbf{u}_{a} \\
\mathbf{X} \boldsymbol{\beta}_{b}+\mathbf{W} \mathbf{c}_{b}+\mathbf{Z} \mathbf{u}_{b}, \mathbf{R} \otimes \mathbf{I} \\
\mathbf{X} \boldsymbol{\beta}_{d}+\mathbf{W} \mathbf{c}_{d}+\mathbf{Z} \mathbf{u}_{d}
\end{array}\right),
\end{aligned}
$$

where $\boldsymbol{\beta}_{\mathrm{a}}, \boldsymbol{\beta}_{\mathrm{b}}$, and $\boldsymbol{\beta}_{\mathrm{d}}$ are vectors of systematic effects on $\mathbf{a}, \mathbf{b}$, and $\mathbf{d}$, respectively; $\mathbf{c}_{\mathrm{a}}, \mathbf{c}_{\mathrm{b}}$, and $\mathbf{c}_{\mathrm{d}}$ are vectors of common litter effects; and $\mathbf{u}_{\mathrm{a}}, \mathbf{u}_{\mathrm{b}}$, and $\mathbf{u}_{\mathrm{d}}$ are vectors of additive genetic effects for the intercept and the partial coefficients of regression, respectively; $\mathbf{X}, \mathbf{W}$, and $\mathbf{Z}$ are incidence matrices; and $\mathbf{R} \otimes \mathbf{I}$ is the (co)variance matrix of the random environmental effects, where $\mathbf{R}$ is the (co)variance matrix between these effects within individual. We thus assumed that the random effects were independent between individuals, but not within each individual.

Third Stage of the Model. The third stage of the model describes the uncertainty about first and second stage parameters (joint prior density). We considered that genetic and common litter effects were independently distributed and that they had a normal prior distribution:

$$
\begin{gathered}
\left(\begin{array}{l}
\mathbf{c}_{a} \\
\mathbf{c}_{b} \\
\mathbf{c}_{d}
\end{array}\right) \quad \mathbf{C}(\mathbf{0}, \mathbf{C} \otimes \mathbf{I}), \text { and } \\
\left(\begin{array}{l}
\mathbf{u}_{a} \\
\mathbf{u}_{b} \\
\mathbf{u}_{d}
\end{array}\right) \sim N(\mathbf{0}, \mathbf{G} \otimes \mathbf{A}),
\end{gathered}
$$

where $\mathbf{G}$ is the genetic (co)variance matrix of the intercept and the partial coefficients of regression, $\mathbf{A}$ is the numerator relationship matrix, and $\mathbf{C}$ is the (co)variance matrix of common litter effects of the intercept and the partial coefficients of regression. We also assumed that the systematic effects and the (co)variance components ( $\mathbf{R}, \mathbf{C}$, and $\mathbf{G}$ ) had uniform prior distributions with boundaries to ensure the posterior distributions to be proper distributions (Press, 1989). 
Residuals in the first-stage of the model were assumed to be independent of the interindividual (second-stage) residuals, given the vector of individual intercepts and partial coefficients of regression. They were also assumed to be independent between individuals; thus, let $\phi^{\prime}=\left\{\boldsymbol{\beta}_{a}, \boldsymbol{\beta}_{b}, \boldsymbol{\beta}_{d}, \mathbf{c}_{a}, \mathbf{c}_{b}, \mathbf{c}_{d}, \mathbf{u}_{a}, \mathbf{u}_{b}, \mathbf{u}_{d}, \mathbf{C}\right.$, $\left.\mathbf{G}, \mathbf{R}, \sigma_{\varepsilon}^{2}\right\}$. The joint prior density was

$$
\begin{gathered}
p(\phi)=p\left(\boldsymbol{\beta}_{a}, \boldsymbol{\beta}_{b}, \boldsymbol{\beta}_{d}\right) \times p\left(\mathbf{c}_{a}, \mathbf{c}_{b}, \mathbf{c}_{d} \mid \mathbf{C}\right) \\
\times p\left(\mathbf{u}_{a}, \mathbf{u}_{b}, \mathbf{u}_{d} \mid \mathbf{G}\right) \times p(\mathbf{C}) \times p(\mathbf{G}) \times p(\mathbf{R}) \times p\left(\sigma_{\varepsilon}^{2}\right) .
\end{gathered}
$$

The joint posterior density was

$$
\begin{gathered}
p\left(\boldsymbol{\theta}, \boldsymbol{\phi} \mid \mathbf{y} \propto p\left(\mathbf{y} \mid \boldsymbol{\theta}, \sigma_{\varepsilon}^{2}\right)\right. \\
\times p\left(\boldsymbol{\theta} \mid \boldsymbol{\beta}_{a}, \boldsymbol{\beta}_{b}, \boldsymbol{\beta}_{d}, \mathbf{c}_{a}, \mathbf{c}_{b}, \mathbf{c}_{d}, \mathbf{u}_{a}, \mathbf{u}_{b}, \mathbf{u}_{d}, \mathbf{C}, \mathbf{G}, \mathbf{R}\right) \times p(\phi), \\
\text { and } p(\boldsymbol{\theta}, \boldsymbol{\phi} \mid \mathbf{y}) \propto p\left(\mathbf{y} \mid \mathbf{a}, \mathbf{b}, \mathbf{d}, \sigma_{\varepsilon}^{2}\right) \\
\times p\left(\mathbf{a}, \mathbf{b}, \mathbf{d} \mid \boldsymbol{\beta}_{a}, \boldsymbol{\beta}_{b}, \boldsymbol{\beta}_{d}, \mathbf{c}_{a}, \mathbf{c}_{b}, \mathbf{c}_{d}, \mathbf{u}_{a}, \mathbf{u}_{b}, \mathbf{u}_{d}, \mathbf{C}, \mathbf{G}, \mathbf{R}\right) \\
\times p\left(\boldsymbol{\beta}_{a}, \boldsymbol{\beta}_{b}, \boldsymbol{\beta}_{d}\right) \times p\left(\mathbf{c}_{a}, \mathbf{c}_{b}, \mathbf{c}_{d} \mid \mathbf{C}\right) \times p\left(\mathbf{u}_{a}, \mathbf{u}_{b}, \mathbf{u}_{d} \mid \mathbf{G}\right) \\
\times p(\mathbf{C}) \times p(\mathbf{G}) \times p(\mathbf{R}) \times p\left(\sigma_{\varepsilon}^{2}\right) .
\end{gathered}
$$

Marginal posterior distributions of all unknowns were estimated using a Gibbs sampling procedure (Casella and George, 1992).

The fully conditional posterior distributions for the intercept and the partial coefficients of regression were obtained from the product of 2 normal distributions:

$$
\begin{gathered}
\mathrm{f}\left(\mathrm{a}_{\mathrm{i}} \mid \mathrm{b}_{\mathrm{i}}, \mathrm{d}_{\mathrm{i}}, \boldsymbol{\theta}_{-\mathrm{i}}, \boldsymbol{\beta}, \mathbf{u}, \mathbf{c}, \sigma_{\varepsilon}^{2}, \mathbf{R}, \mathbf{y}\right) \propto \mathrm{f}\left(\mathbf{y}_{\mathrm{i}} \mid \mathrm{a}_{\mathrm{i}}, \mathrm{b}_{\mathrm{i}}, \mathrm{d}_{\mathrm{i}}, \sigma_{\varepsilon}^{2}\right) \\
\times \mathrm{f}\left(\mathrm{a}_{\mathrm{i}} \mid \mathrm{b}_{\mathrm{i}}, \mathrm{d}_{\mathrm{i}}, \boldsymbol{\beta}, \mathbf{u}, \mathbf{c}, \mathbf{R}\right) .
\end{gathered}
$$

1) From the conditional distribution of the data:

$$
\sim \mathrm{N}\left[\frac{\mathrm{a}_{\mathrm{i}} \mid \mathrm{b}_{\mathrm{i}}, \mathrm{d}_{\mathrm{i}}, \sigma_{\varepsilon}^{2}, \mathbf{y}_{\mathrm{i}}}{\sum_{j=1}^{n_{i}}\left(y_{i j}-b_{i} \times A B W_{i j}^{0.75}-d_{i} \times B W G_{i j}\right)}, \frac{\sigma_{\varepsilon}^{2}}{n_{i}}\right],
$$

$$
\begin{aligned}
& \mathrm{b}_{\mathrm{i}} \mid \mathrm{a}_{\mathrm{i}}, \mathrm{d}_{\mathrm{i}}, \sigma_{\varepsilon}^{2}, \mathbf{y}_{\mathrm{i}} \sim
\end{aligned}
$$

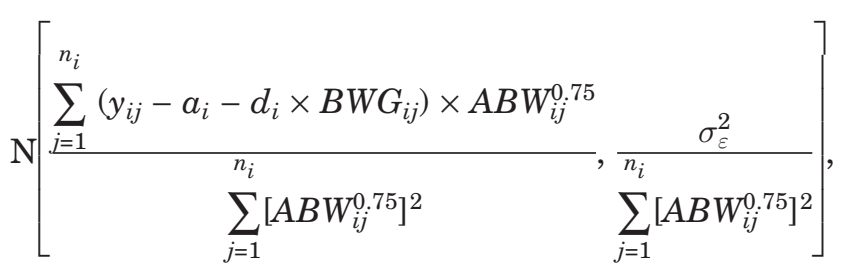

$$
\begin{aligned}
& \text { and } d_{i} \mid a_{i}, b_{i}, \sigma_{\varepsilon}^{2}, \mathbf{y}_{i} \\
& \sim \mathrm{N}\left[\frac{\sum_{j=1}^{n_{i}\left(y_{i j}-a_{i}-b_{i} \times A B W_{i j}^{0.75}\right) \times B W G_{i j}}}{\sum_{j=1}^{n_{i}}\left[B W G_{i j}\right]^{2}}, \frac{\sigma_{\varepsilon}^{2}}{\sum_{j=1}^{n_{i}}\left[B W G_{i j}\right]^{2}}\right] .
\end{aligned}
$$

2) From the conditional distribution of the intercept and both coefficients of regression given genetic and environmental effects:

$$
\begin{gathered}
\mathrm{a}_{\mathrm{i}} \mid \mathrm{b}_{\mathrm{i}}, \mathrm{d}_{\mathrm{i}}, \boldsymbol{\beta}, \mathbf{u}, \mathbf{c}, \mathbf{R} \\
\sim N\left(m_{a}-\frac{r^{a b}}{r^{a a}}\left(b_{i}-m_{b}\right)-\frac{r^{a d}}{r^{a a}}\left(d_{i}-m_{d}\right), 1 / r^{a a}\right), \\
\sim N\left(m_{b}-\frac{r^{a b}}{r^{b b}}\left(a_{i}-\mathbf{a}_{\mathrm{i}}, \mathbf{d}_{\mathrm{i}}, \beta, \mathrm{u}, \mathrm{c}, \mathrm{R}\right.\right. \\
\left.r^{b b}\left(d_{i}-m_{d}\right), 1 / r^{b b}\right), \text { and } \\
\sim N\left(m_{d}-\frac{r^{a d}}{r^{d d}}\left(a_{i}-m_{a}\right)-\frac{r^{b d}}{r^{d d}}\left(b_{i}-m_{b}\right), 1 / \mathbf{a}^{d d}\right),
\end{gathered}
$$

where $\mathrm{r}^{\mathrm{aa}}, \mathrm{r}^{\mathrm{ab}}, \mathrm{r}^{\mathrm{ad}}, \mathrm{r}^{\mathrm{bb}}, \mathrm{r}^{\mathrm{bd}}, \mathrm{r}^{\mathrm{dd}}$ are the corresponding elements of the inverse of residual (co)variance matrix (R); and $\mathrm{m}_{\mathrm{a}}, \mathrm{m}_{\mathrm{b}}, \mathrm{m}_{\mathrm{d}}$ the corresponding means for $\mathrm{a}, \mathrm{b}$, d given $\boldsymbol{\beta}, \mathbf{u}, \mathbf{c}$, and $\mathbf{R}$ :

$$
\begin{gathered}
m_{a}=\mathbf{X}_{i} \boldsymbol{\beta}_{a}+\mathbf{W}_{i} \mathbf{c}_{a}+\mathbf{Z}_{i} \mathbf{u}_{a}, \\
m_{b}=\mathbf{X}_{i} \boldsymbol{\beta}_{b}+\mathbf{W}_{i} \mathbf{c}_{b}+\mathbf{Z}_{i} \mathbf{u}_{b}, \text { and } \\
m_{d}=\mathbf{X}_{i} \boldsymbol{\beta}_{d}+\mathbf{W}_{i} \mathbf{c}_{d}+\mathbf{Z}_{i} \mathbf{u}_{d} .
\end{gathered}
$$

The fully conditional posterior distribution for the location parameters was

$$
\begin{gathered}
\mathrm{f}\left(\boldsymbol{\beta}, \mathbf{u}, \mathbf{c} \mid \boldsymbol{\theta}, \sigma_{\varepsilon}^{2}, \mathbf{G}, \mathbf{C}, \mathbf{R}, \mathbf{y}\right) \propto \mathrm{f}\left(\boldsymbol{\theta} \mid \boldsymbol{\beta}, \mathbf{u}, \mathbf{c}, \sigma_{\varepsilon}^{2}, \mathbf{G}, \mathbf{C}, \mathbf{R}, \mathbf{y}\right) \\
\times \mathrm{f}(\boldsymbol{\beta}) \times \mathrm{f}(\mathbf{u} \mid \mathbf{G}) \times \mathrm{f}(\mathbf{c} \mid \mathbf{C})
\end{gathered}
$$

Thus,

$$
\mathrm{g}_{\mathrm{i}} \mid \mathbf{g}_{-\mathrm{i}}, \boldsymbol{\theta}, \sigma_{\varepsilon}^{2}, \mathbf{G}, \mathbf{C}, \mathbf{R}, \mathbf{y} \sim \mathrm{N}\left[\frac{R H S_{i}-\sum_{i \neq j} l_{i j} g_{i}}{l_{i j}}, \frac{1}{l_{i j}}\right],
$$

where $\mathbf{g}_{-i}$ is the vector $\mathbf{g}^{\prime}=\left[\boldsymbol{\beta}^{\prime}, \mathbf{u}^{\prime}, \mathbf{c}^{\prime}\right]$ without the element i, $R H S_{i}$ is the corresponding element of the Right-Hand Side, and $l_{i j}$ the corresponding coefficient of the mixed model equations constructed as if the observed traits were the intercept and the regression coefficients:

$$
\begin{gathered}
{\left[\begin{array}{ccc}
\mathbf{X}^{\prime} \mathbf{R}^{-1} \mathbf{X} & \mathbf{X}^{\prime} \mathbf{R}^{-1} \mathbf{Z} & \mathbf{X}^{\prime} \mathbf{R}^{-1} \mathbf{W} \\
\mathbf{Z}^{\prime} \mathbf{R}^{-1} \mathbf{X} & \mathbf{Z}^{\prime} \mathbf{R}^{-1} \mathbf{Z}+(\mathbf{G} \otimes \mathbf{A})^{-1} & \mathbf{Z}^{\prime} \mathbf{R}^{-1} \mathbf{W} \\
\mathbf{W}^{\prime} \mathbf{R}^{-1} \mathbf{X} & \mathbf{W}^{\prime} \mathbf{R}^{-1} \mathbf{Z} & \mathbf{W}^{\prime} \mathbf{R}^{-1} \mathbf{W}+(\mathbf{I} \otimes \mathbf{C})^{-1}
\end{array}\right]} \\
{\left[\begin{array}{c}
\boldsymbol{\beta} \\
\mathbf{u} \\
\mathbf{c}
\end{array}\right]=\left[\begin{array}{c}
\mathbf{X}^{\prime} \mathbf{R}^{-1} \boldsymbol{\theta} \\
\mathbf{Z}^{\prime} \mathbf{R}^{-1} \boldsymbol{\theta} \\
\mathbf{W}^{\prime} \mathbf{R}^{-1} \boldsymbol{\theta}
\end{array}\right] .}
\end{gathered}
$$

The fully conditional posterior distributions for the (co)variance matrices were 
$\mathrm{f}\left(\mathbf{R} \mid \boldsymbol{\theta}, \sigma_{\varepsilon}^{2}, \boldsymbol{\beta}, \mathbf{u}, \mathbf{c}, \mathbf{y}\right) \propto \mathrm{f}(\boldsymbol{\theta} \mid \boldsymbol{\beta}, \mathbf{u}, \mathbf{c}, \mathbf{R}) \times \mathrm{f}(\mathbf{R})$.

Thus,

$$
\begin{gathered}
\mathbf{R} \mid \boldsymbol{\theta}, \sigma_{\varepsilon}^{2}, \boldsymbol{\beta}, \mathbf{u}, \mathbf{c}, \mathbf{y} \\
\sim W^{-1}\left(N-4,(\boldsymbol{\theta}-\mathbf{X} \boldsymbol{\beta}-\mathbf{Z u}-\mathbf{W} \mathbf{c})^{\prime}(\boldsymbol{\theta}-\mathbf{X} \boldsymbol{\beta}-\mathbf{Z u}-\mathbf{W} \mathbf{c})\right) \\
\mathrm{f}\left(\mathbf{G} \mid \boldsymbol{\theta}, \sigma_{\varepsilon}^{2}, \boldsymbol{\beta}, \mathbf{u}, \mathbf{c}, \mathbf{y}\right) \propto \mathrm{f}(\boldsymbol{\theta} \mid \boldsymbol{\beta}, \mathbf{u}, \mathbf{c}, \mathbf{G}) \times \mathrm{f}(\mathbf{G}) .
\end{gathered}
$$

Thus,

$$
\mathbf{G} \mid \boldsymbol{\theta}, \sigma_{\varepsilon}^{2}, \boldsymbol{\beta}, \mathbf{u}, \mathbf{c}, \mathbf{y} \sim W^{-1}\left(q-4, \mathbf{U}^{\prime} \mathbf{A}^{-1} \mathbf{U}\right)
$$

where $\mathrm{U}=\left[\mathbf{u}_{\mathrm{a}}, \mathbf{u}_{\mathrm{b}}, \mathbf{u}_{\mathrm{d}}\right]$, where $\mathbf{u}_{\mathrm{a}}, \mathbf{u}_{\mathrm{b}}$, and $\mathbf{u}_{\mathrm{d}}$ are vectors with the genetic values of the intercept and regression coefficients for all individuals of the genealogy, and $q$ is the number of individuals in the genealogy. In addition,

$$
\mathrm{f}\left(\mathbf{C} \mid \boldsymbol{\theta}, \sigma_{\varepsilon}^{2}, \boldsymbol{\beta}, \mathbf{u}, \mathbf{c}, \mathbf{y}\right) \propto \mathrm{f}(\boldsymbol{\theta} \mid \boldsymbol{\beta}, \mathbf{u}, \mathbf{c}, \mathbf{C}) \times \mathrm{f}(\mathbf{C}) .
$$

Thus,

$$
\mathbf{C} \mid \boldsymbol{\theta}, \sigma_{\varepsilon}^{2}, \boldsymbol{\beta}, \mathbf{u}, \mathbf{c}, \mathbf{y} \sim \mathrm{W}^{-1}\left(\mathrm{p}-4, \mathbf{P}^{\prime} \mathbf{P}\right),
$$

where $\mathbf{P}^{\prime}=\left[\mathbf{c}_{\mathrm{a}}^{\prime}, \mathbf{c}_{\mathrm{b}}^{\prime}, \mathbf{c}_{\mathrm{d}}^{\prime}\right] . \mathbf{c}_{\mathrm{a}}, \mathbf{c}_{\mathrm{b}}$, and $\mathbf{c}_{\mathrm{d}}$ are vectors with the common litter effects of the intercept and regression coefficients, and $\mathrm{p}$ is the number of levels of this factor.

The fully conditional posterior distribution for fitting the error variance was

$$
\mathrm{f}\left(\sigma_{\varepsilon}^{2} \mid \boldsymbol{\theta}, \boldsymbol{\beta}, \mathbf{u}, \mathbf{c}, \mathbf{R}, \mathbf{y}\right) \propto \mathrm{f}\left(\mathbf{y} \mid \boldsymbol{\theta}, \sigma_{\varepsilon}^{2}\right) .
$$

Thus,

$$
\begin{gathered}
\sigma_{\varepsilon}^{2} \mid \boldsymbol{\theta}, \boldsymbol{\beta}, \mathbf{u}, \mathbf{c}, \mathbf{R}, \mathbf{y} \\
\sim \chi^{-2}\left[\sum _ { i = 1 } ^ { N } \sum _ { j = 1 } ^ { n _ { i } } \left[y_{i j}-a_{i}-b_{i} \times A B W_{i j}^{0.75}\right.\right. \\
\left.\left.-d_{i} \times B W G_{i j}\right]^{2}, N_{d}-2\right],
\end{gathered}
$$

where $\mathrm{N}_{\mathrm{d}}$ was the number of observations.

\section{Implementation}

The procedure was implemented using data from 2 elliptical selection experiments performed to estimate the genetic parameters of BW gain and feed conversion ratio during the fattening period in rabbit lines Caldes and $R$. The elliptical selection design (Cameron and Thompson, 1986) is based on selecting parents with extreme values and estimating the heritability by offspring-parent regression. The extreme individuals are the animals that lie outside of an ellipse defined by a
Table 1. Number of records and means (SD) for individual metabolic BW (MBW, g $\mathrm{g}^{0.75} / 7 \mathrm{~d}$ ) and BW gain (BWG, $\mathrm{g} / 7 \mathrm{~d}$ ) in each $7-\mathrm{d}$ period, in the Caldes and $\mathrm{R}$ lines of rabbits

\begin{tabular}{lcccc}
\hline \hline Line & Neriod & $\begin{array}{c}\text { No. of } \\
\text { rabbits }\end{array}$ & $M B W$ & $B W G$ \\
\hline Caldes & 1 & 433 & $177(29)$ & $362(81)$ \\
& 2 & 439 & $225(34)$ & $405(80)$ \\
& 3 & 439 & $273(37)$ & $397(75)$ \\
$\mathrm{R}$ & 4 & 437 & $315(38)$ & $349(71)$ \\
& 1 & 443 & $188(26)$ & $410(69)$ \\
& 2 & 442 & $240(30)$ & $426(75)$ \\
& 3 & 437 & $290(31)$ & $421(64)$ \\
& 4 & 434 & $333(33)$ & $337(79)$ \\
\hline
\end{tabular}

quadratic index, $I_{i}=\mathbf{x}_{i}^{\prime} \mathbf{P}_{0}^{-1} \mathbf{x}_{i}$, where $\mathbf{x}_{\mathrm{i}}$ is the 2-trait data vector of individual $\mathrm{i}$, and $\mathbf{P}_{0}$ is the phenotypic (co)variance matrix of these traits.

The Caldes line was formed by crossing animals from 5 New Zealand White lines and a California $\times$ New Zealand synthetic line. Line $\mathrm{R}$ originated by crossing a California line with a synthetic line created by mating rabbits from commercial male lines. Both lines were selected for growth rate by individual selection from 1993 and 1980, respectively (Estany et al., 1992; Gómez et al., 1999).

Experimental animals were allocated to individual cages on the experimental farm of IRTA and were fed ad libitum with a commercial diet $(16.4 \% \mathrm{CP}, 15.2 \%$ fiber, $4 \%$ fat, DM basis). Body weight (g) and cumulative feed intake (FI, g) during the fattening period (from weaning at $32 \mathrm{~d}$ of age to $60 \mathrm{~d}$ of age) were recorded weekly. Individual $\mathrm{BW}$ gain (BWG, g) and average BW (g) were calculated from these data per animal for each 7-d period and for the entire fattening period. The correlation between MBW and BWG was low in both lines $(0.22$ and 0.05 in the Caldes and $R$ lines, respectively). The number of animals measured was 444 and 445 in the Caldes and $\mathrm{R}$ lines, respectively.

The means (SD) of the individual metabolic BW and BW gain in each period and each line are shown in Table 1. Details about the experimental design, the elliptical selection procedure, and the intensity of selection from weighted selection differentials are given in Piles et al. (2003).

The statistical analysis was performed for each line separately. The increase in residual variance due to a scale effect was considered to be negligible because the fattening period is very short in rabbits (only $4 \mathrm{wk}$ ). On the other hand, the number of observations was small and it was necessary to reduce the number of parameters to estimate. Thus, residuals were considered to be homoscedastic and independently distributed. A uniform prior distribution was also considered for the individual intercepts, instead of the probability density coming from the second stage of the hierarchi- 
cal model. Thus, the joint posterior density for the analyses was

$$
\begin{gathered}
p(\boldsymbol{\theta}, \boldsymbol{\phi} \mid \mathbf{y}) \propto p\left(\mathbf{y} \mid \mathbf{a}, \mathbf{b}, \mathbf{d}, \sigma_{\varepsilon}^{2}\right) \\
\times p\left(\mathbf{b}, \mathbf{d} \mid \boldsymbol{\beta}_{b}, \boldsymbol{\beta}_{d}, \mathbf{c}_{b}, \mathbf{c}_{d}, \mathbf{u}_{b}, \mathbf{u}_{d}, \mathbf{C}, \mathbf{G}, \mathbf{R}\right) \times p(\mathbf{a}) \\
\times p\left(\boldsymbol{\beta}_{b}, \boldsymbol{\beta}_{d}\right) \times p\left(\mathbf{c}_{b}, \mathbf{c}_{d} \mid \mathbf{C}\right) \times p\left(\mathbf{u}_{b}, \mathbf{u}_{d} \mid \mathbf{G}\right) \times p(\mathbf{C}) \\
\times p(\mathbf{G}) \times p(\mathbf{R}) \times p\left(\sigma_{\varepsilon}^{2}\right) .
\end{gathered}
$$

The model assumed for each coefficient of regression included the systematic effects of: sex (male or female), batch (10 levels), litter size in which the animal was born (8 levels: $<6,6,7,8,9,10,11$, and $>11$ ), parity order (3 levels: first, second, third, and greater), the common litter effect, and the additive genetic effect. There were 561 and 553 animals in the pedigree file for the Caldes and $\mathrm{R}$ lines, respectively, corresponding to all of the animals from the elliptical selection experiments and their parents.

The Gibbs sampler was implemented using 3 chains of 2,500,000 iterations. The first 500,000 iterations of each chain were discarded, and samples of the parameters of interest were saved for each of 10 iterations. Gibbs samples were used to estimate features of the marginal posterior distribution (i.e., mean, SD, and posterior credibility regions of size $95 \%$ ). Gelman and Rubin's (1992) diagnostic test was used to assess convergence. Convergence was also tested for each chain using the criteria of Geweke (1992). Autocorrelation between samples and Monte-Carlo error of features of marginal distributions (Geyer, 1992) were also calculated.

\section{RESULTS AND DISCUSSION}

In order to evaluate the goodness of fit of the model and the adequacy of some of the assumptions made, we plotted the means of the posterior distributions of residuals against the means of the posterior distributions of the predicted values in both lines (Figure 1). This plot reveals that there was no dependence of the residuals on the predicted values and the assumption of constant residual variance held.

Table 2 shows the summary statistics of the estimated marginal posterior distributions of the proportion of the phenotypic variance due to additive genetic effects $\left(h^{2}\right)$, the proportion of the phenotypic variance due to common litter effects $\left(c^{2}\right)$, and phenotypic variance $\left(\sigma^{2}\right)$ for partial coefficients of regression of feed intake on metabolic BW (b) and feed intake on BW gain $(d)$, and the residual variance $\left(\sigma_{\varepsilon}^{2}\right)$, in the Caldes and $\mathrm{R}$ lines. Table 2 also shows the values of several features of the chains that describe the simulation processes. From visual inspection of the trace plots and results of Geweke's test, there was no evidence against convergence for each variable in each simulation process. Moreover, the value of the scale factor of the Gelman and Rubin test was 1 or very close to 1
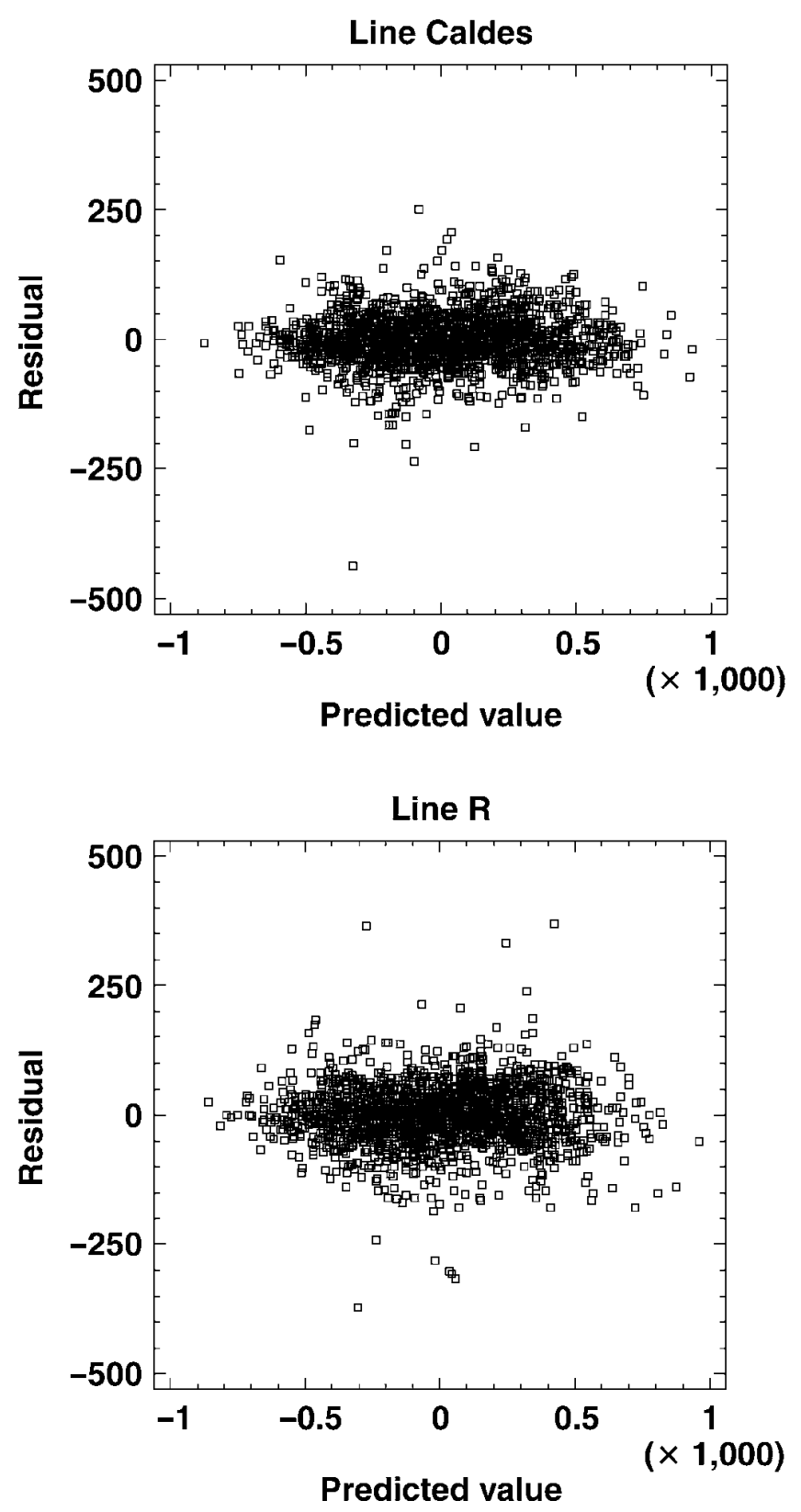

Figure 1. Plot of the means of the posterior distributions of residuals against the means of the posterior distributions of the predicted feed intake $(\mathrm{g} / \mathrm{d})$ in the Caldes and $\mathrm{R}$ lines of rabbits.

(the lowest value was 0.99 ) for all the parameters in both lines. The autocorrelations between successive samples of the same chain were high, indicating poor mixing and small effective sample size. However, pooling samples over chains after convergence, the Monte Carlo SE were in all cases smaller than $0.4 \%$ of the posterior mean, which suggests that the simulation processes were sufficiently long and that the estimates of features of the marginal posterior distributions could be considered sufficiently accurate.

Estimated marginal posterior distributions were nearly symmetrical and approximated a normal distri- 
Table 2. Heritability $\left(\mathrm{h}^{2}\right)$, proportion of the phenotypic variance due to common litter effects $\left(c^{2}\right)$, and phenotypic variance $\left(\sigma^{2}\right)$ for partial coefficients of regression of feed intake $(\mathrm{g} / 7 \mathrm{~d})$ on metabolic BW $\left(\mathrm{g}^{0.75} / 7 \mathrm{~d}\right)$ and feed intake $(\mathrm{g} / 7 \mathrm{~d})$ on BW gain $(\mathrm{g} / 7 \mathrm{~d})$, and residual variance $\left(\sigma_{\varepsilon}^{2}\right)$ in the Caldes and $\mathrm{R}$ lines of rabbits

\begin{tabular}{|c|c|c|c|c|c|c|c|}
\hline Line & $\mathrm{P}^{1}$ & Item & $\mathrm{PM}^{2}$ & $\mathrm{PSD}^{3}$ & HPD $95 \%^{4}$ & $\mathrm{r}^{5}$ & $\mathrm{MCse}^{6}$ \\
\hline \multirow[t]{7}{*}{ Caldes } & \multirow[t]{3}{*}{$\mathrm{b}$} & $\mathrm{h}^{2}$ & 0.348 & 0.171 & {$[0.011,0.653]$} & 0.95 & 0.00099 \\
\hline & & $c^{2}$ & 0.389 & 0.136 & {$[0.128,0.650]$} & 0.86 & 0.00069 \\
\hline & & $\sigma^{2}$ & 0.350 & 0.064 & {$[0.229,0.474]$} & 0.69 & 0.00024 \\
\hline & \multirow[t]{4}{*}{$\mathrm{d}$} & $\mathrm{h}^{2}$ & 0.404 & 0.169 & {$[0.095,0.729]$} & 0.94 & 0.00095 \\
\hline & & $c^{2}$ & 0.275 & 0.130 & {$[0.042,0.521]$} & 0.86 & 0.00062 \\
\hline & & $\sigma^{2}$ & 0.286 & 0.060 & {$[0.178,0.405]$} & 0.78 & 0.00026 \\
\hline & & $\sigma_{\varepsilon}^{2}$ & 4,373 & 208 & {$[3,968,4,786]$} & 0.13 & 0.398 \\
\hline \multirow[t]{7}{*}{$\mathrm{R}$} & \multirow[t]{3}{*}{$\mathrm{b}$} & $\mathrm{h}^{2}$ & 0.259 & 0.189 & {$[0.0003,0.633]$} & 0.98 & 0.00101 \\
\hline & & $c^{2}$ & 0.436 & 0.221 & {$[0.0008,0.811]$} & 0.97 & 0.00116 \\
\hline & & $\sigma^{2}$ & 0.066 & 0.029 & {$[0.018,0.123]$} & 0.95 & 0.00015 \\
\hline & \multirow[t]{4}{*}{$\mathrm{d}$} & $\mathrm{h}^{2}$ & 0.268 & 0.140 & {$[0.032,0.533]$} & 0.96 & 0.00073 \\
\hline & & $c^{2}$ & 0.492 & 0.136 & {$[0.227,0.746]$} & 0.90 & 0.00066 \\
\hline & & $\sigma^{2}$ & 0.347 & 0.077 & {$[0.207,0.500]$} & 0.82 & 0.00034 \\
\hline & & $\sigma_{\varepsilon}^{2}$ & 5,540 & 243 & {$[5,074,6,023]$} & 0.13 & 0.453 \\
\hline
\end{tabular}

${ }^{1} \mathrm{P}=$ parameter: $\mathrm{b}$, partial coefficient of regression of feed intake $(\mathrm{g} / 7 \mathrm{~d})$ on metabolic $\mathrm{BW}\left(\mathrm{g}^{0.75} / 7 \mathrm{~d}\right) ; \mathrm{d}$, partial coefficient of regression of feed intake $(\mathrm{g} / 7 \mathrm{~d})$ on $\mathrm{BW}$ gain $(\mathrm{g} / 7 \mathrm{~d})$; and $\sigma_{\varepsilon}^{2}$, residual variance.

${ }^{2} \mathrm{PM}=$ posterior mean.

${ }^{3} \mathrm{PSD}=$ posterior $\mathrm{SD}$.

${ }^{4} \mathrm{HPD} 95 \%$ = highest posterior density of $95 \%$.

${ }^{5} \mathrm{r}=$ autocorrelation between successive samples of the same chain.

${ }^{6} \mathrm{MCse}=$ Monte Carlo SE.

bution; thus, the SD provided a good indication of the accuracy of the estimates. The marginal posterior means (SD) of genetic variance were $0.121(0.063)$ and 0.118 (0.059) for $b$ and $d$, respectively, in the Caldes line and $0.017(0.015)$ and $0.092(0.051)$ for $b$ and $d$, respectively, in line $R$. The values of the limit $k$ in the interval from $k$ to infinity that contains $95 \%$ of the area of the marginal posterior density were 0.03 and 0.04 for $b$ and $d$, respectively, in the Caldes line and, 0.002 and 0.02 for $b$ and $d$, respectively, in line $R$. The marginal posterior means of the proportion of the phenotypic variance due to additive genetic effects of partial coefficients of regression were moderate, but due to the small amount of data, the SD of the posterior density for these parameters was very high. The values of the limit $k$ in the interval from $k$ to infinity that contains $95 \%$ of the area of the marginal posterior density were 0.08 and 0.14 for $b$ and $d$, respectively, in the Caldes line and, 0.03 and 0.07 for $b$ and $d$, respectively, in line $R$. These results suggest that it is feasible to improve efficiency of animals for the use of feed resources through individual predictions of the additive genetic values for these parameters. The partial coefficient of regression of feed intake on metabolic BW represents maintenance requirements per kilogram of metabolic weight, whereas the partial coefficient of regression of feed intake on BW gain is related to the efficiency of use of resources for growth. It should be noted that some bias could exist in the estimates of the partial regression coefficients and variance components because both populations had been selected for growth rate during the fattening period and the entire history of the selection procedure was not con- tained in the data employed for analysis (Gianola and Fernando, 1986).

The proportion of environmental effects related to the litter was not negligible for the 2 coefficients of regression in both lines (Table 2). The estimated posterior means (posterior SD) were $0.39(0.14)$ and 0.28 (0.13) for $b$ and $d$, respectively, in the Caldes line and $0.44(0.22)$ and $0.49(0.14)$ for $b$ and $d$, respectively, in line $\mathrm{R}$. The greater importance of these effects during the fattening period, compared with other species such as pigs, is due to the proportionately shorter interval of time from weaning to slaughter. In rabbits this interval is only $4 \mathrm{wk}$, whereas in pigs, for example, it is approximately 5 mo.

Posterior mean and posterior SD of the phenotypic and genetic correlations between the partial coefficients of regression in the Caldes and $\mathrm{R}$ lines are shown in Table 3. Due to the small amount of data, the estimates of those parameters were very inaccurate and nothing can be known about the genetic relationship between the 2 partial regression coefficients and the possible correlated response in the other trait when a population is selected for increased efficiency of feed use for growth or against feed required for BW maintenance.

Inferences with the proposed model are conditional to the values of BWG and MBW. Both traits, BWG and MBW, are subject to genetic and environmental variation, and, therefore, more complex models could be proposed to avoid the possible bias in the estimates of the partial regression coefficients and variance components. These models include both a recursive (Gianola and Sorensen, 2004) and a reaction norm model 
Table 3. Phenotypic $\left(r_{p}\right)$ and genetic $\left(r_{g}\right)$ correlations between partial coefficients of regression of feed intake $(\mathrm{g} / 7 \mathrm{~d})$ on metabolic BW $\left(\mathrm{g}^{0.75} / 7 \mathrm{~d}\right)$ and feed intake $(\mathrm{g} / 7 \mathrm{~d})$ on BW gain $(\mathrm{g} / 7 \mathrm{~d})$ in the Caldes and $\mathrm{R}$ lines of rabbits

\begin{tabular}{lllccrr}
\hline \hline Line & $\mathrm{P}^{1}$ & $\mathrm{PM}^{2}$ & $\mathrm{PSD}^{3}$ & HPD 95\% & $\mathrm{r}^{5}$ & $\mathrm{MCse}^{6}$ \\
\hline Caldes & $\mathrm{r}_{\mathrm{g}}$ & 0.515 & 0.446 & {$[-0.336,0.991]$} & 0.98 & 0.0027 \\
& $\mathrm{r}_{\mathrm{p}}$ & 0.093 & 0.144 & {$[-0.187,0.372]$} & 0.78 & 0.0006 \\
$\mathrm{R}$ & $\mathrm{r}_{\mathrm{g}}$ & 0.599 & 0.532 & {$[-0.738,1.00]$} & 0.99 & 0.0114 \\
& $\mathrm{r}_{\mathrm{p}}$ & 0.699 & 0.171 & {$[0.364,0.972]$} & 0.95 & 0.0068 \\
\hline
\end{tabular}

\footnotetext{
${ }^{1} \mathrm{P}=$ parameter.

${ }^{2} \mathrm{PM}=$ posterior mean.

${ }^{3} \mathrm{PSD}=$ posterior $\mathrm{SD}$.

${ }^{4} \mathrm{HPD} 95 \%$ = highest posterior density of $95 \%$.

${ }^{5} \mathrm{r}=$ autocorrelation between successive samples of the same chain.

${ }^{6} \mathrm{MCse}=$ Monte Carlo SE.
}

(Su et al., 2006), with very complex implementation and identification problems. These models will be an interesting topic for future research.

The model proposed could be extended to include coefficients of regression of feed consumption on other production traits, such as backfat thickness in pigs or egg production in poultry. Moreover, the model could have some interesting applications in research of the genetic causes of obesity and their relationship with feed intake and energy expenditure. The model can easily be adapted to include QTL or gene effects, and, as a consequence, it can be used to study the role of some regions of the genome, such as the leptin, leptin receptor, or peroxisome proliferator-activated receptor-gamma genes (Paracchini et al., 2005) in the allocation of feed resources in livestock or human populations.

A procedure based on the hierarchical Bayesian scheme and on multiple regression models for the analysis of individual feed resource allocation pattern was described and applied to data from animals of 2 sire lines of rabbits. The procedure allows estimation of systematic effects and variance components, and prediction of breeding values for the use of feed resources for maintenance of metabolic BW and BW gain, which can be used in a selection index to improve the global efficiency of use of feed resources. Estimated heritabilities of partial coefficients of regression were moderate to high in the 2 populations, indicating that efficiency of feed use could be improved by selecting for these coefficients. However, the accuracy of these estimates was low and more research is needed with a larger data set to draw reliable conclusions about the efficiency of selection and the correlated responses. The proposed model could be extended to include other production traits.

\section{LITERATURE CITED}

Armero, E., and A. Blasco. 1992. Economic weights for rabbit selection indices. J. Appl. Rabbit Res. 15:637-642.

Blasco, A. 2001. The Bayesian controversy in animal breeding. J. Anim. Sci. 79:2023-2046.
Blasco, A., M. Piles, and L. Varona. 2003. A Bayesian analysis of the effect of selection for growth rate on growth curves in rabbits. Genet. Sel. Evol. 35:21-45.

Cameron, N. D., and R. Thompson. 1986. Design of multivariate selection experiments to estimate genetic parameters. Theor. Appl. Genet. 72:466-476.

Casella, G., and E. I. George. 1992. Explaining the Gibbs Sampler. Am. Stat. 46:167-174.

Estany, J., J. Camacho, M. Baselga, and A. Blasco. 1992. Selection response of growth rate in rabbits for meat production. Genet. Sel. Evol. 24:527-537.

Gelman, A., and D. B. Rubin. 1992. Inference from iterative simulation using multiple sequences. Stat. Sci. 7:457-472.

Geweke, J. 1992. Evaluating the accuracy of sampling-based approaches to the calculation of posterior moments (with discussion). Pages 169-193 in Bayesian Statistics 4, J. M. Bernardo, J. O. Berger, A. P. Dawid, and A. F. M. Smith, ed. Oxford University Press, Oxford, UK.

Geyer, C. J. 1992. Practical Markov Chain Monte Carlo. Stat. Sci. 7:473-511.

Gianola, D., and R. L. Fernando. 1986. Random effects models for binary responses. Biometrics 42:217-218.

Gianola, D., and D. Sorensen. 2004. Quantitative genetic models for describing simultaneous and recursive relationships between phenotypes. Genetics 167:1407-1424.

Gómez, E. A., O. Rafel, J. Ramon, and M. Baselga. 1999. Selection, diffusion and performances of six Spanish lines of meat rabbit. Options Mèditerr., Sèr, Cah 147-152.

Mignon-Gastreau, S., C. Beaumont, E. Le Bihan-Duval, J. P. Poivey, H. de Rochambeau, and F. H. Ricard. 2000. Genetic parameters of growth curve parameters in male and female chickens. Br. Poult. Sci. 40:44-51.

Paracchini, V., P. Pedotti, and E. Taioli. 2005. Genetics of leptin and obesity: A HuGE review. Am. J. Epidemiol. 162:101-114.

Piles, M., D. Gianola, L. Varona, and A. Blasco. 2003. Bayesian inference about parameters of a longitudinal trajectory when selection operates on a correlated trait. J. Anim. Sci. 81:2714-2724

Piles, M., E. A. Gómez, O. Rafel, J. Ramon, and A. Blasco. 2004. Elliptical selection experiment for the estimation of genetic parameters of the growth rate and feed conversion ratio in rabbits. J. Anim. Sci. 82:654-660.

Press, S. J. 1989. Bayesian Statistics: Principles, Models and Applications. John Wiley and Sons, New York, NY.

Rauw, W. M., P. Luiting, M. W. A. Verstegen, O. Vangen, and P. W. Knap. 2000. Differences in food resource allocation in a longterm selection experiment for litter size in mice. II. Developmental trends in BW against food intake. Anim. Sci. 71:39-47.

Rauw, W. M., P. W. Knap, M. W. A. Verstegen, and P. Luiting. 2002. Food resource allocation patterns in lactating females in a longterm selection experiment for litter size in mice. Genet. Sel. Evol. 34:83-104. 
Rekaya, R., M. J. Carabaño, and M. A. Toro. 2000. Bayesian analysis of lactation curves of Holstein-Friesian cattle using a nonlinear model. J. Dairy Sci. 83:2691-2701.

Rekaya, R., K. A. Weigel, and D. Gianola. 2001. Hierarchical nonlinear model for persistency of milk yield in the first three lactations of Holsteins. Livest. Prod. Sci. 68:181-187.

Su, G., P. Madsen, M. S. Lund, D. Sorensen, I. R. Korsgaard, and J. Jensen. 2006. Bayesian analysis of the linear reaction norm model with unknown covariates. J. Anim. Sci. 84:1651-1657.
Varona, L., C. Moreno, L. A. García-Cortés, and J. Altarriba. 1997. Multiple trait genetic analysis of underlying biological variables of production functions. Livest. Prod. Sci. 47:201-209.

Varona, L., C. Moreno, L. A. García-Cortés, and J. Altarriba. 1998. Bayesian analysis of the Wood's lactation curve for Spanish dairy cows. J. Dairy Sci. 81:1469-1478.

Wakefield, J. C., A. F. M. Smith, A. Racine-Poon, and A. E. Gelfand. 1994. Bayesian analysis of linear and non-linear population models by using the Gibbs sampler. Appl. Stat. 43:2201-2221. 